\title{
EFFICACY OF COMPLEMENTARY AND ALTERNATIVE MEDICINE IN TREATMENT OF POSTPARTUM DEPRESSION: A SITUATION ANALYSIS
}

\author{
Bakhtawar M.Hanif Khowaja* \& Ghulam Kubra Rind \\ Department of Obstetrics and Gynecology, The Aga Khan University, Stadium Road, Karachi , \\ Pakistan
}

${ }^{*}$ Corresponding author: bakhtawarshalwani92@gmail.com

\begin{abstract}
Postpartum depression is a major public health issue worldwide. It varies in prevalence across countries. Pakistan remains highest to have the prevalence of postpartum depression among South Asian countries. A number of complementary and alternative medicines can help in decreasing depressive symptoms in the postpartum period. The purpose of this article is to subject review the use of complementary and alternative medicine as a treatment for postpartum depression. A comprehensive review of the literature was conducted from articles using PubMed, CINAHL, PsycINFO, EMBASE published since the last ten years. The findings of this review suggest that various complementary and alternative therapies may help in the treatment of postpartum depression. This literature review demonstrated the significant effectiveness of complementary and alternative medicine in the treatment of postpartum depression. As conclusion, the prevention and treatment of postpartum depression are essential for maternal as well as new-born health. Complementary and alternative medicine has less side effects than antidepressants which can affect maternal and newborn health adversely.
\end{abstract}

Keywords: Postpartum depression; complementary and alternative medicine; Pakistan. 


\section{Introduction}

\section{Postpartum depression}

Postpartum depression, also called postnatal depression, is a type of mood disorder associated with childbirth. It is a major depressive disorder affecting $6.5-30 \%$ of postnatal women(Stewart \& Vigod, 2016). The symptoms of postpartum depression include extreme grief, low-energy, anxiety, crying, irritability, and changes in sleeping or eating patterns(Starr, 2017). The onset of PPD is usually between 7 days to 40 days following childbirth(Kettunen \& Hintikka, 2017). The exact cause of PPD is not clear and includes a combination of factors like physical, emotional, genetic, and social factors (Stewart \& Vigod, 2016) The hormonal changes in women following childbirth is identified to be a major cause of PPD (Trifu, Vladuti, Popescu , 2019). The treatment for PPD is important to decrease the risk of chronic depression in postnatal mothers. Postpartum depression was first identified as a disorder in the $16^{\text {th }}$ century (Ghaedrahmati,Kazemi, Kheirabadi, et al, 2017). Since then, experimental treatments started as implementation for PPD. In the $20^{\text {th }}$ century, it was included under the umbrella of mental illnesses. (Starr, 2017)

Postpartum depression is a major public health issue worldwide. It varies in prevalence across countries (Prabhu, George, Shyamala, et al, 2019). Evidence suggests that the global burden of PPD is around $17.7 \%$ (Stewart \& Vigod, 2016). The Low-Middle Income countries have the highest prevalence of PPD. (Azale, Fekadu, \& Hanlon, 2018). Countries like Singapore, Denmark, and Malaysia report a low prevalence of postpartum depression (0.5-9\%), whereas, in countries like South Africa, Taiwan, and Korea, there is a high prevalence of PPD (34-57\%) (Halbreich \& Karkun, 2006). The high prevalence of PPD in low-income countries is due to the low investment of these countries on maternal and mental health (Dadi, Miller \& Mwanri, 2020). Most of the women with PPD in lowincome countries remain undetected due to ignored mental health and nearly $80 \%$ of women with PPD remain undiagnosed (Learman, 2018).

The prevalence of PPD in South Asia ranges from 3.5\% to 63.3\%(Aliani \& Khuwaja, 2017). Pakistan remains highest to have the prevalence of PPD among South Asian countries and accounts for PPD from $28 \%$ to $63 \%$. (Aliani \& Khuwaja, 2017) The health care expenditure on mental health in Pakistan is only $0.4 \%(\mathrm{Naaz}$, Mehnaz, Ansari, \& Amir, 2020). Moreover, maternal health is also neglected in Pakistan and the abandoned maternal and mental health in Pakistan increases the risk of PPD in the country. The major risk factors for postpartum depression in Pakistan include sociocultural factors involving gender bias, female mobility issues, postpartum customs and rituals, sociocultural traditions and the extended family system(Naaz et al., 2020). 
The treatment for PPD comprises a range of antidepressants(McCloskey \& Reno, 2019). The American Academy of Pediatrics considers antidepressants as a concern of postpartum treatment given to breastfeeding mothers. Antidepressants can have negative effects on breastfeeding infants(Bandoli, Chambers, Wells, \& Palmsten, 2020). A number of complementary and alternative medicines can help in decreasing depressive symptoms in the postpartum period.

Complementary and alternative medicine refers to therapies that range beyond conventional medical treatment. The term complementary refers to the treatment used as a combination with standard care and the term alternative refers to the therapies used as a replacement, other than standard conventional medications(Weber \& Hopp, 2020). There are several CAM therapies including dietary supplements, massage, yoga, exercise, and acupuncture that can help in the treatment of postpartum depression(Weber \& Hopp, 2020). The purpose of this article is to subject review the approach of complementary and alternative medicine as a treatment for postpartum depression.

\section{Methods}

A comprehensive review of literature for the subject review was conducted from articles using PubMed, CINAHL, PsycINFO, EMBASE using terms such as Postpartum depression, Pakistan, South Asia, complementary and alternative medicine, yoga, exercise, dietary supplementation, acupuncture, etcetera. Moreover, links to "related articles" were accessed in electronic databases. Inclusion criteria included only full-text papers in the English language published in the last ten years were preferred. Research studies were selected that included risk factors of PPD, CAM therapies, and CAM therapies for depression, especially in Pakistan.

\section{Results}

The results of the literature review suggested that National Center for Complementary and Integrative Health defines complementary health approaches in two categories as natural products and mind/body practices(Weber \& Hopp, 2020). Natural products include herbal medicines, probiotics, minerals, and vitamins. Mind and body practices involve a diverse group of techniques including acupuncture, massage therapy, meditation, relaxation therapies, and yoga(Kinser, Robins, \& Jallo, 2021). The findings of this review suggest that various complementary and alternative therapies may help in the treatment of postpartum depression.

\section{Natural Products}

According to National Center for Complementary and Integrative Health $(\mathrm{NCClH})$, natural health products include (e.g. omega-3 fatty acids), dietary supplements, probiotics, vitamins and minerals, botanicals, and herbal remedies(Dennis, Brown, \& Brown, 2019). The natural products have been proven to provide benefits in the treatment of postpartum depression. 


\section{Nutraceuticals}

Nutraceuticals are defined as non-prescribed natural health products. They are naturally occurring substances that include minerals, and vitamins (e.g. (acetyl-L-carnitine), tryptophan, inositol, folic acid, amino acids, alpha-lactalbumin, dehydroepiandrosterone (DHEA), omega-3 fatty acids, and SAdenosylmethionine (SAM-e). (Ravindran et al., 2018) They can be used alone or in combination with anti-depressants for the treatment of postpartum depression.

\section{Omega-3 Fatty Acids}

Omega 3 fatty acids are polyunsaturated fatty acids that originated from biological systems. They are formed of docosahexaenoic acid (DHA), or cetyl esters of eicosapentaenoic acid (EPA), or a combination of both. Omega 3 fatty acids are involved in enhancing the serotonergic neurotransmission, regulation of corticotrophin-releasing factor, and changes that are related to the dopamine function. (LaChance \& Ramsey, 2018) however, the mechanism of action requires more research to identify its role in the improvement of depression and stress (Häberling, Berger, Schmeck, Held, \& Walitza, 2019). One of the pilot trials found that women experiencing PPD had significant improvement by receiving Omega-3 fatty acid intervention and had a reduction in their depressive symptoms. (Mermer \& Şanlıer) A cross-sectional study reported that depressive disorders in women are mostly associated with low consumption of omega 3 fatty acids mostly found in seafood (Young et al., 2017). Several randomized controlled trials have been conducted that confirm Omega 3 fatty acids to have a greater impact on depressive disorders. (Nasab, Pakseresht, \& Behbahani, 2019). There have been no short-term side-effects related to consumption of omega 3 fatty acids reported, however the long-term supplementation can cause increased risk for cancer due to oxidation products and added Vitamin E (Lange, Nakamura, Gosslau \& Li, 2019).

\section{Folate}

Folate is comprised of the one-carbon cycle and it works by modifying the production of neurotransmitters including serotonin, dopamine, and norepinephrine that reduces the depressive symptoms. (Ng, Nazir, \& Nault, 2020) A study on Iranian women was conducted to determine the efficiency of Iron supplementation among women who had undergone for $C$ section. $37.1 \%$ of mothers with PPD were found to have iron deficiency without anemia. A significant association was found with increase iron intake and decline in the Edinburgh Postnatal Depression Scale and $42.8 \%$ improvement in PPD. (Naaz et al., 2020).

\section{Vitamin-D}

Evidence suggests that Vitamin-D is found to have a significant effect on brain development and its functioning. In the Central Nervous Systems, there are special nuclear receptors for 1, 25-dihydroxy vitamin $D$ along with vital enzymes for hydroxylation of vitamin $D$. Thus, the brain activates vitamin $D$ that leads to the prominence of vitamin D role in brain activities. (Bahrami et al., 2018) It has been reported that a low level of 25-hydroxyvitamin $D$ is linked to increased psychological symptoms including anxiety, depression, and decreased cognition. (Gallone et al., 2017) Evidence suggests that 
consumption of vitamin $D_{3}$ during late pregnancy is useful in reducing the post-partum depression level among women. Another study was found to have significant results of the high dose of vitamin $D$ among men and women who were depressed.(Berlanga-Taylor et al., 2017)

\section{Herbal remedies}

Herbs are derivates from plants and their extracts and include leaves, roots, flowers, berries, and bark. Examples of herbs include Crocus sativus, Lavandula angustifolia, St. John's wort (Hypericum perforatum), and Rhodiola Rosea.(Raheel, Orabi, \& El-Masry, 2019) It has been reported that herbs alone or in combination provide a significant antidepressant effect as they control the neurotransmitters monoamine, inflammatory cytokines, and T- cell mediators immune response(Lee \& Bae, 2017). One of the studies conducted in Iran showed that daily consumption of $30 \mathrm{mg}$ Saffron decreased the severity of depression with no significant adverse outcome on the breastfeeding mothers(Tabeshpour et al., 2017). However, few studies suggest the negative effects of herbs on embryos if taken during pregnancy (Esmaeilzadeh \& Moradi, 2017).

\section{Mind and body practices}

Mind and body practices are considered the most common complementary health practices and are used to treat conditions like depression, anxiety, and stress. Evidence suggests that more than $50 \%$ of people suffering from anxiety and depression, use some type of complementary health approach. These techniques can lift and energize the body(Fulweiler \& John, 2018). Several mind and body practices are found to be effective in improving postpartum depression. These approaches have been discussed following.

\section{Acupuncture}

Considering the neuroendocrine disorder theory of depression, acupuncture reduces depressive symptoms by exerting effects through the hypothalamus-adrenal axis and hypothalamus-pituitarythyroid axis(Crowley et al., 2018). Acupuncture regulates the status of postpartum depression by improving hormone levels. Several researches have been conducted to evaluate the effects of acupuncture on depression. A randomized controlled trial for the treatment of depression, conducted by University of York researchers found that there was a significant reduction in depressive symptoms with acupuncture without any adverse effects (Li et al., 2019). Acupuncture is effective as well as safe for patients with depression. Another randomized controlled trial held in one of the hospitals from China concluded acupuncture with psychological intervention as effective as antidepressants without adverse effects for postpartum depression(Zhang et al., 2020).

\section{Massage therapy}

Massage therapy helps in relieving physical distress. It improves blood circulation and slows down the production of the stress hormone, cortisol. One of the studies on the effects of massage found massage therapy in the reduction of cortisol level by $31 \%$ (Nahayati et al., 2020). Some studies on the effects of massage therapy confirm reducing depressive symptoms. Massage therapy helps in increasing relaxation, pain relief, and sleep and in reducing swelling during the postpartum period. 
Massage therapy during pregnancy is proven to be beneficial for mothers in the postnatal period (Haseli, Ghiasi, \& Hashemzadeh, 2019). One of the studies demonstrated the effects of massage therapy during pregnancy on mothers and newborns. The newborns of these mothers have healthier birth weights and reduced cortisol levels. (Álvarez et al., 2017) Evidence suggests that massage therapy is not only beneficial to those diagnosed with postpartum depression, it also helps as a prevention to develop postpartum depression.

\section{Mindfulness}

Mindfulness is effective in reducing postpartum depression as it provides mothers with awareness and information about negative events such as sleep disorders, stress, and burdens. Evidence suggests that mindfulness helps mothers to control negative feelings(Azadi, Manshaee, \& Golparvar, 2019). As hormonal changes during pregnancy and childbirth are considered to be a major cause of postpartum depression, mindfulness helps mothers in reducing depression by increasing awareness about psychological conditions. Mindfulness facilitates mothers in the development of their coping strategies, in reducing impulsivity, and in reducing anxiety in postnatal mothers. (Müller et al., 2020) Several studies have shown prayer to be effective in reducing depressive symptoms and anxiety (Johnson et al. 2009). Centering prayer may hold promising results as an alternative intervention for improvement in chronic depression.

\section{$\underline{\text { Yoga }}$}

Yoga is an ancient practice that originated from India. It is derived from a Sanskrit word that means "to yoke or to unite". Postpartum mothers usually gain weight following childbirth and have poor psychological functioning due to changes in hormonal levels. Yoga can help in the improvement of physical activity as well as psychological benefits that can help postpartum mothers. Yoga helps in the improvement of conditions like agitation and anxiety through mindfulness. Yoga provides antidepressive effects and promotes a sense of self-efficacy(Müller et al., 2020). Research evidence suggests the benefits of yoga in chronic health conditions including pain, stress, and insomnia. It also provides useful effects during labor pain and depression. (Jahdi et al., 2017)

\section{$\underline{\text { Music }}$}

Music therapy is known to reduce anxiety, stress and depression. According to quantitative research, many patients in hospital settings who were suffering from stress, anxiety, pain, and fear of death, were able to relieve their negative emotions with the help of music therapy (Västfjäll, Juslin, \& Hartig, 2012). Clinical application of music therapy on clients with mental illnesses can have an intense effect on restoration. According to a survey by Nizamie \& Tikka (2014), music therapy showed $45 \%$ effectiveness in clients with mental illnesses. When compared to antipsychotics, its side-effect profile was extremely safe whereas the latter developed $25 \%$ harmful side-effects in patients with mental diseases. Thus, music therapy can decrease antipsychotics side-effects in patients if used in combination with pharmacological treatment. 


\section{Discussion}

This literature review revealed the significant effectiveness of CAM in the treatment of PPD. A number of studies including cross-sectional studies and randomized controlled trials have explored the role of natural products and mind/body practices in the treatment of postpartum depression. These studies have been conducted mostly in high-income countries. There is a nonexistence of researches on complementary and alternative therapies in South Asian countries especially in Pakistan where there is a high prevalence of postpartum depression. Complementary and alternative therapies provide safe treatment as an alternative to antidepressants and provide better efficacy. These therapies not only provide treatment for postpartum depression but can also be used to prevent risks of developing postpartum depression.

\section{Conclusion}

CAM therapies including natural products and mind/body practices have demonstrated promising results in the prevention and treatment of postpartum depression. Research evidence indicates that these effects have important clinical implications and they need to be studied further to implement these therapies in clinical settings. Moreover, the health policy implications should be developed by health care authorities and providers to prevent postpartum depression. The prevention and treatment of postpartum depression are essential for maternal as well as new-born health.

- A number of complementary and alternative medicines can help in decreasing depressive symptoms in the postpartum period.

- Complementary and alternative therapies provide safe treatment as an alternative to antidepressants and provide better efficacy. These therapies not only provide treatment for postpartum depression but they can also be used to prevent risks of developing postpartum depression.

- Research evidence indicates that these effects have important clinical implications and they need to be studied further especially in LMICs like Pakistan to implement these therapies in clinical settings where there is high burden of PPD.

\section{Abbreviations}

- CAM: Complementary and Alternative medicine; DHEA: dehydroepiandrosterone; NNCIH: National Center for Complementary and Integrative Health; PPD: Postpartum depression; SAM-e: S-Adenosylmethionine

\section{Conflicts of Interest:}

The authors declare no conflicts of interest. 


\section{References}

- Aliani, R., \& Khuwaja, B. (2017). Epidemiology of postpartum depression in Pakistan: A review of literature. National Journal of Health Sciences, 2, 24-30.

- Álvarez, M. J., Fernández, D., Gómez-Salgado, J., Rodriguez-Gonzalez, D., Rosón, M., \& Lapena, S. (2017). The effects of massage therapy in hospitalized preterm neonates: A systematic review. International journal of nursing studies, 69, 119-136.

- Azadi, M. M., Manshaee, G., \& Golparvar, M. (2019). Comparing the Effectiveness of Mobile Social Network-Based Mindfulness Interventions with Acceptance and Commitment Therapy (ACT) and Mindfulness Therapy on Depression, Anxiety, Stress among Patients with Type 2 Diabetes. Journal of Diabetes Nursing, 7(2), 775-792.

- Azale, T., Fekadu, A., \& Hanlon, C. (2018). Postpartum depressive symptoms in the context of high social adversity and reproductive health threats: a population-based study. International journal of mental health systems, 12(1), 1-10.

- Bahrami, A., Mazloum, S. R., Maghsoudi, S., Soleimani, D., Khayyatzadeh, S. S., Arekhi, S., . . Bahrami-Taghanaki, H. (2018). High dose vitamin D supplementation is associated with a reduction in depression score among adolescent girls: a nine-week follow-up study. Journal of dietary supplements, 15(2), 173-182.

- Bandoli, G., Chambers, C. D., Wells, A., \& Palmsten, K. (2020). Prenatal antidepressant use and risk of adverse neonatal outcomes. Pediatrics, 146(1).

- Berlanga-Taylor, A. J., Plant, K., Dahl, A., Lau, E., Hill, M., Sims, D., . . Clarke, R. (2017). Effect of vitamin $D$ supplementation on biomarkers of inflammation and immune function: functional genomics analysis of the BEST-D trial. BioRxiv, 217612.

- Crowley, M. J., Nicholls, S. S., McCarthy, D., Greatorex, K., Wu, J., \& Mayes, L. C. (2018). Innovations in practice: group mindfulness for adolescent anxiety-results of an open trial. Child and Adolescent Mental Health, 23(2), 130-133.

- Dadi, A. F., Miller, E. R., \& Mwanri, L. (2020). Postnatal depression and its association with adverse infant health outcomes in low-and middle-income countries: a systematic review and meta-analysis. BMC pregnancy and childbirth, 20(1), 1-15.

- Dennis, C. L., Brown, J. V. E., \& Brown, H. K. (2019). Interventions (other than psychosocial, psychological and pharmacological) for treating postpartum depression. The Cochrane Database of Systematic Reviews, 2019(11).

- Esmaeilzadeh, M., \& Moradi, B. (2017). Medicinal herbs with side effects during pregnancyAn evidence-based review article. The Iranian Journal of Obstetrics, Gynecology and Infertility, 20(ن ويزه), 9-25.

- Fulweiler, B., \& John, R. M. (2018). Mind \& body practices in the treatment of adolescent anxiety. The nurse practitioner, 43(8), 36-43.

- Gallone, G., Haerty, W., Disanto, G., Ramagopalan, S. V., Ponting, C. P., \& Berlanga-Taylor, A. J. (2017). Identification of genetic variants affecting vitamin D receptor binding and associations with autoimmune disease. Human molecular genetics, 26(11), 2164-2176.

- Ghaedrahmati, M., Kazemi, A., Kheirabadi, G., Ebrahimi, A., \& Bahrami, M. (2017). Postpartum depression risk factors: A narrative review. Journal of education and health promotion, 6.

- Häberling, I., Berger, G., Schmeck, K., Held, U., \& Walitza, S. (2019). Omega-3 Fatty Acids as a Treatment for Pediatric Depression. A Phase III, 36 Weeks, Multi-Center, Double-Blind, Placebo-Controlled Randomized Superiority Study. Frontiers in psychiatry, 10, 863.

- Halbreich U, Karkun S. Cross-cultural and social diversity of prevalence of postpartum depression and depressive symptoms. Journal of affective disorders. 2006 Apr 1;91(2-3):97111.

- Haseli, A., Ghiasi, A., \& Hashemzadeh, M. (2019). Do Breathing Techniques Enhance the effect of massage therapy in reducing the length of labor or not? a randomized clinical trial. Journal of caring sciences, 8(4), 257.

- Jahdi, F., Sheikhan, F., Haghani, H., Sharifi, B., Ghaseminejad, A., Khodarahmian, M., \& Rouhana, N. (2017). Yoga during pregnancy: The effects on labor pain and delivery outcomes (A randomized controlled trial). Complementary therapies in clinical practice, 27, 1-4.

- Nizamie, S. H., \& Tikka, S. K. (2014). Psychiatry and music. Indian journal of psychiatry, 56(2), 128.Johnson, M., Dose, A., Pipe, T., Petersen, W., Huschka, M., 
Gallenberg, M., et al. (2009). Centering prayer for women receiving chemotherapy for recurrent ovarian cancer: A pilot study. Oncology Nursing Forum, 36, 421-428.

- Kettunen, P., \& Hintikka, J. (2017). Psychosocial risk factors and treatment of new onset and recurrent depression during the post-partum period. Nordic journal of psychiatry, 71(5), 355361.

- Kinser, P. A., Robins, J. L. W., \& Jallo, N. (2021). Complementary and Integrative Health Approaches for Women's Mental Health. In Integrative Health Nursing Interventions for Vulnerable Populations (pp. 157-167): Springer.

- LaChance, L. R., \& Ramsey, D. (2018). Antidepressant foods: An evidence-based nutrient profiling system for depression. World journal of psychiatry, 8(3), 97.

- Lange, K. W., Nakamura, Y., Gosslau, A. M., \& Li, S. (2019). Are there serious adverse effects of omega-3 polyunsaturated fatty acid supplements?. Journal of Food Bioactives, 7.

- Learman, L. A. (2018). Screening for depression in pregnancy and the postpartum period. Clinical obstetrics and gynecology, 61(3), 525-532.

- Lee, G., \& Bae, H. (2017). Therapeutic effects of phytochemicals and medicinal herbs on depression. BioMed research international, 2017.

- Li, M., Xing, X., Yao, L., Li, X., He, W., Wang, M., . . . Yan, P. (2019). Acupuncture for treatment of anxiety, an overview of systematic reviews. Complementary therapies in medicine, 43, 247-252.

- McCloskey, R. J., \& Reno, R. (2019). Complementary health approaches for postpartum depression: A systematic review. Social Work in Mental Health, 17(1), 106-128.

- Müller, M., Matthies, L. M., Goetz, M., Abele, H., Brucker, S. Y., Bauer, A., . . . Wallwiener, M. (2020). Effectiveness and cost-effectiveness of an electronic mindfulness-based intervention $(\mathrm{eMBI})$ on maternal mental health during pregnancy: the mindmom study protocol for a randomized controlled clinical trial. Trials, 21(1), 1-11.

- Naaz, N., Mehnaz, S., Ansari, M. A., \& Amir, A. (2020). MATERNAL MENTAL HEALTH AND ITS ASSOCIATION WITH SOCIODEMOGRAPHIC FACTORS AND DELIVERY RELATED PRACTICES. Global Journal For Research Analysis (GJRA), 9(6).

- Nahayati, M. A., Vaghar Seyyedin, S. A., Bahrami-Taghanki, H. R., Rezaee, Z., Mehrpooya, N., \& Rahimi, H. (2020). Effect of Acupressure on Stress and Anxiety of Patients with Multiple Sclerosis: A Sham-controlled Randomized Clinical Trial. Complementary Medicine Journal, 10(3), 270-283.

- Nasab, M. N., Pakseresht, S., \& Behbahani, S. (2019). The Effect of adding Non-saturated fatty acid Omega 3 and Placebo on anti-depressant drugs among patients with depression disorder. Journal of Advanced Pharmacy Education \& Research| Apr-Jun, 9(S2), 155.

- Ng, J. Y., Nazir, Z., \& Nault, H. (2020). Complementary and alternative medicine recommendations for depression: a systematic review and assessment of clinical practice guidelines. BMC complementary medicine and therapies, 20(1), 1-15.

- Prabhu, S., George, L. S., Shyamala, G., Noronha, J. A., \& Hebbar, S. (2019). Prevalence and Associated Risk Factors of Postnatal Depression in South Asian Region--A Systematic Review. Indian Journal of Public Health Research \& Development, 10(5).

- Raheel, I., Orabi, A., \& El-Masry, A. (2019). Natural herbs CLEANACTIV®; Immunemodulator, health activator and growth promoter in broiler chickens. International Journal of Veterinary Science, 8(4), 267-270.

- Ravindran, A. V., Balneaves, L. G., Faulkner, G., Ortiz, A., Mclntosh, D., Morehouse, R. L., . . . Lam, R. W. (2018). Canadian network for mood and anxiety treatments (CANMAT) 2016 clinical guidelines for the management of adults with major depressive disorder: section 5 . complementary and alternative medicine treatments. Focus, 16(1), 85-94.

- Starr, R. F. (2017). Beyond the Baby Blues: Anxiety and Depression During and After Pregnancy: Rowman \& Littlefield.

- Stewart, D. E., \& Vigod, S. (2016). Postpartum depression. New England Journal of Medicine, 375(22), 2177-2186.

- Tabeshpour, J., Sobhani, F., Sadjadi, S. A., Hosseinzadeh, H., Mohajeri, S. A., Rajabi, O., . . . Eslami, S. (2017). A double-blind, randomized, placebo-controlled trial of saffron stigma (Crocus sativus L.) in mothers suffering from mild-to-moderate postpartum depression. Phytomedicine, 36, 145-152.

- Trifu, S., Vladuti, A., \& Popescu, A. (2019). The neuroendocrinological aspects of pregnancy and postpartum depression. Acta Endocrinologica (Bucharest), 15(3), 410.Weber, W. J., \& Hopp, D. C. (2020). National Center for Complementary and Integrative Health Perspectives 
on Clinical Research Involving Natural Products. Drug Metabolism and Disposition, 48(10), 963-965.

- Västfjäll, D., Juslin, P. N., \& Hartig, T. (2012). Music, subjective wellbeing, and health: The role of everyday emotions. Music, health \& wellbeing, 405-423.

- Young, A. J., Marriott, B. P., Champagne, C. M., Hawes, M. R., Montain, S. J., Johannsen, N. M., . . . Hibbeln, J. R. (2017). Blood fatty acid changes in healthy young Americans in response to a 10-week diet that increased n-3 and reduced n-6 fatty acid consumption: a randomised controlled trial. British Journal of Nutrition, 117(9), 1257-1269.

- Zhang, L., Tang, Y., Hui, R., Zheng, H., Deng, Y., Shi, Y., . . Yu, S. (2020). The effects of active acupuncture and placebo acupuncture on insomnia patients: a randomized controlled trial. Psychology, health \& medicine, 25(10), 1201-1215. 